International Journal of Pure and Applied Mathematics

Volume 106 No. 1 2016, 87-99

ISSN: 1311-8080 (printed version); ISSN: 1314-3395 (on-line version)

url: http://www.ijpam.eu

doi: 10.12732/ijpam.v106i1.7

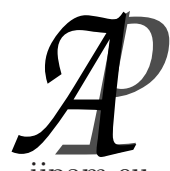

ijpam.eu

\title{
COMMON FIXED POINTS FOR WEAKLY COMPATIBLE \\ MAPPINGS SATISFYING BINARY OPERATIONS \\ IN MULTIPLICATIVE METRIC SPACES
}

\author{
Parveen Kumar ${ }^{1}$, Sanjay Kumar ${ }^{2}$, Shin Min Kang ${ }^{3} \S$ \\ ${ }^{1,2}$ Department of Mathematics \\ Deenbandhu Chhotu Ram University of Science and Technology \\ Murthal, Sonepat 131039, Haryana, INDIA \\ ${ }^{3}$ Department of Mathematics and RINS \\ Gyeongsang National University \\ Jinju 52828, KOREA
}

\begin{abstract}
In this paper, we prove some common fixed point theorems for weakly compatible mappings satisfying binary operations in multiplicative metric spaces.
\end{abstract}

AMS Subject Classification: 47H10, 54H25

Key Words: multiplicative metric space, weakly compatible mapping, binary operation, multiplicative $\alpha$-property

\section{Introduction and Preliminaries}

It is well know that the set of positive real numbers $\mathbb{R}_{+}$is not complete according to the usual metric. To overcome this problem, in 2008, Bashirov et al. [3] introduced the concept of multiplicative metric spaces as follows:

Definition 1.1. Let $X$ be a nonempty set. A multiplicative metric is a mapping $d: X \times X \rightarrow \mathbb{R}_{+}$satisfying the following conditions:

(i) $d(x, y) \geq 1$ for all $x, y \in X$ and $d(x, y)=1$ if and only if $x=y$;

(ii) $d(x, y)=d(y, x)$ for all $x, y \in X$;

Received: August 14, 2015

Published: February 2, 2016

$\S$ Correspondence author
(C) 2016 Academic Publications, Ltd.

url: www.acadpubl.eu 
(iii) $d(x, y) \leq d(x, z) \cdot d(z, y)$ for all $x, y, z \in X$ (multiplicative triangle inequality).

Then the mapping $d$ together with $X$, that is, $(X, d)$ is a multiplicative metric space.

Example 1.2. ([9]) Let $\mathbb{R}_{+}^{n}$ be the collection of all $n$-tuples of positive real numbers. Let $d^{*}: \mathbb{R}_{+}^{n} \times \mathbb{R}_{+}^{n} \rightarrow \mathbb{R}$ be defined as follows:

$$
d^{*}(x, y)=\left|\frac{x_{1}}{y_{1}}\right|^{*} \cdot\left|\frac{x_{2}}{y_{2}}\right|^{*} \cdots\left|\frac{x_{n}}{y_{n}}\right|^{*}
$$

where $x=\left(x_{1}, \ldots, x_{n}\right), y=\left(y_{1}, \ldots, y_{n}\right) \in \mathbb{R}_{+}^{n}$ and $|\cdot|^{*}: \mathbb{R}_{+} \rightarrow \mathbb{R}_{+}$is defined by

$$
|a|^{*}= \begin{cases}a & \text { if } a \geq 1 \\ \frac{1}{a} & \text { if } a<1\end{cases}
$$

Then it is obvious that all conditions of a multiplicative metric are satisfied. Therefore $\left(\mathbb{R}_{+}^{n}, d\right)$ is a multiplicative metric space.

Example 1.3. ([11]) Let $d: \mathbb{R} \times \mathbb{R} \rightarrow[1, \infty)$ be defined as $d(x, y)=a^{|x-y|}$, where $x, y \in \mathbb{R}$ and $a>1$. Then $d$ is a multiplicative metric and $(X, d)$ is a multiplicative metric space. We may call it usual multiplicative metric spaces.

Remark 1.4. We note that the Example 1.2 is valid for positive real numbers and Example 1.3 is valid for all real numbers.

Example 1.5. ([11]) Let $(X, d)$ be a metric space. Define a mapping $d_{a}$ on $X$ by

$$
d_{a}(x, y)=a^{d(x, y)}= \begin{cases}1 & \text { if } x=y \\ a & \text { if } x \neq y\end{cases}
$$

where $x, y \in X$ and $a>1$. Then $d_{a}$ is a multiplicative metric and $\left(X, d_{a}\right)$ is known as the discrete multiplicative metric space.

Example 1.6. ([1]) Let $X=C^{*}[a, b]$ be the collection of all real-valued multiplicative continuous functions on $[a, b] \subset \mathbb{R}_{+}$. Then $(X, d)$ is a multiplicative metric space with $d$ defined by $d(f, g)=\sup _{x \in[a, b]}\left|\frac{f(x)}{g(x)}\right|$ for arbitrary $f, g \in X$.

Remark 1.7. ([11]) We note that multiplicative metrics and metric spaces are independent.

Indeed, the mapping $d^{*}$ defined in Example 1.2 is multiplicative metric but 
not metric as it does not satisfy triangular inequality. Consider

$$
d^{*}\left(\frac{1}{3}, \frac{1}{2}\right)+d^{*}\left(\frac{1}{2}, 3\right)=\frac{3}{2}+6=7.5<9=d^{*}\left(\frac{1}{3}, 3\right) .
$$

On the other hand the usual metric on $\mathbb{R}$ is not multiplicative metric as it doesnt satisfy multiplicative triangular inequality, since

$$
d(2,3) \cdot d(3,6)=3<4=d(2,6)
$$

One can refer to $[7,9]$ for detailed multiplicative metric topology.

Definition 1.8. Let $(X, d)$ be a multiplicative metric space. Then a sequence $\left\{x_{n}\right\}$ in $X$ said to be

(1) a multiplicative convergent to $x$ if for every multiplicative open ball $B_{\epsilon}(x)=\{y \mid d(x, y)<\epsilon\}, \epsilon>1$, there exists $N \in \mathbb{N}$ such that $x_{n} \in B_{\epsilon}(x)$ for all $n \geq N$, that is, $d\left(x_{n}, x\right) \rightarrow 1$ as $n \rightarrow \infty$.

(2) a multiplicative Cauchy sequence if for all $\epsilon>1$, there exists $N \in \mathbb{N}$ such that $d\left(x_{n}, x_{m}\right)<\epsilon$ for all $m, n \geq N$, that is, $d\left(x_{n}, x_{m}\right) \rightarrow 1$ as $n, m \rightarrow \infty$.

(3) We call a multiplicative metric space complete if every multiplicative Cauchy sequence in it is multiplicative convergent to $x \in X$.

Remark 1.9. The set of positive real numbers $\mathbb{R}_{+}$is not complete according to the usual metric. Let $X=\mathbb{R}_{+}$and the sequence $\left\{x_{n}\right\}=\left\{\frac{1}{n}\right\}$. It is obvious $\left\{x_{n}\right\}$ is a Cauchy sequence in $X$ with respect to usual metric and $X$ is not a complete metric space, since $0 \notin \mathbb{R}_{+}$. In case of a multiplicative metric space, we take a sequence $\left\{x_{n}\right\}=\left\{a^{\frac{1}{n}}\right\}$, where $a>1$. Then $\left\{x_{n}\right\}$ is a Cauchy sequence since for $n \geq m$,

$$
\begin{aligned}
d\left(x_{n}, x_{m}\right) & =\left|\frac{x_{n}}{x_{m}}\right|=\left|\frac{a^{\frac{1}{n}}}{a^{\frac{1}{m}}}\right|=\left|a^{\frac{1}{n}-\frac{1}{m}}\right| \\
& \leq a^{\frac{1}{m}-\frac{1}{n}}<a^{\frac{1}{m}}<\epsilon \text { if } m>\frac{\log a}{\log \epsilon},
\end{aligned}
$$

where $|a|=\left\{\begin{array}{ll}a & \text { if } a \geq 1, \\ \frac{1}{a} & \text { if } a<1 .\end{array}\right.$ Also, $\left\{x_{n}\right\} \rightarrow 1$ as $n \rightarrow \infty$ and $1 \in \mathbb{R}_{+}$. Hence $(X, d)$ is a complete multiplicative metric space.

In 2012, Özavsar and Çevikel [9] gave the concept of multiplicative contraction mappings and proved some fixed point theorem of such mappings in a multiplicative metric space. 
Definition 1.10. Let $f$ be a mapping of a multiplicative metric space $(X, d)$ into itself. Then $f$ is said to be a multiplicative contraction if there exists a real number $\lambda \in[0,1)$ such that

$$
d(f x, f y) \leq d^{\lambda}(x, y) \quad \text { for all } x, y \in X .
$$

Gu et al. [6] introduced the notion of commutative and weak commutative mappings in a multiplicative metric space and proved some fixed point theorems for these mappings.

Definition 1.11. Let $f$ and $g$ be two mappings of a multiplicative metric space $(X, d)$ into itself. Then $f$ and $g$ are said to be

(1) commutative mappings if $f g x=g f x$ for all $x \in X$.

(2) weak commutative mappings if $d(f g x, g f x) \leq d(f x, g x)$ for all $x \in X$.

Notice that commuting mappings are obviously weakly commuting. However, the converse need not be true.

In 1996, Jungck [8] introduced the concept of weakly compatible mappings and prove fixed point theorems using these mappings in metric spaces (see $[2,4,5,10])$.

Now, we introduce the notions in multiplicative metric spaces.

Definition 1.12. Let $f$ and $g$ be two mappings of a multiplicative metric space $(X, d)$ into itself. Then $f$ and $g$ are said to be weakly compatible if they commute at coincidence points, that is, $f t=g t$ for some $t \in X$ implies that $f g t=g f t$.

Notice that weakly commuting mappings are obviously weakly compatible. However, the converse need not be true.

In 2007, Sedghi and Shobe [12] introduced a new binary operation $\diamond$ as follows:

Let $\diamond: \mathbb{R}_{+} \times \mathbb{R}_{+} \rightarrow \mathbb{R}_{+}$be a binary operation satisfying

(i) $\diamond$ is associative and commutative,

(ii) $\diamond$ is continuous.

Some examples of a binary operation $\diamond$ are as follows: for each $a, b \in \mathbb{R}_{+}$,

(1) $a \diamond b=\max \{a, b\}$

(2) $a \diamond b=a+b$,

(3) $a \diamond b=a b$,

(4) $a \diamond b=a b+a+b$,

(5) $a \diamond b=\frac{a b}{\max \{a, b, 1\}}$. 
Definition 1.13. The binary operation $\diamond$ is said to satisfy $\alpha$-property if there exists a positive real number $\alpha$ such that $a \diamond b \leq \alpha \max \{a, b\}$ for all $a, b \in \mathbb{R}_{+}$.

Example 1.14. (1) If $a \diamond b=a+b$ for each $a, b \in \mathbb{R}_{+}$, then for $\alpha \geq 2$, we have $a \diamond b \leq \alpha \max \{a, b\}$.

(2) If $a \diamond b=a b$ for each $a, b \in \mathbb{R}_{+}$, then for $\alpha \geq \min \{a, b\}$, we have $a \diamond b \leq \alpha \max \{a, b\}$.

(3) If $a \diamond b=\frac{a b}{\max \{a, b, 1\}}$ for each $a, b \in \mathbb{R}_{+}$, then for $\alpha \geq 1$, we have $a \diamond b \leq$ $\alpha \max \{a, b\}$.

Now we define $\alpha$-property in a multiplicative metric space sense as follows:

Definition 1.15. The binary operation $\diamond$ is said to satisfy a multiplicative $\alpha$-property if there exists a positive real number $\alpha$ such that $a \diamond b \leq(\max \{a, b\})^{\alpha}$ for all $a, b \in \mathbb{R}_{+}$.

Example 1.16. (1) If $a \diamond b=a b$ for each $a, b \in \mathbb{R}_{+}$, then for $\alpha \geq 2$, we have $a \diamond b \leq(\max \{a, b\})^{\alpha}$.

(2) If $a \diamond b=\frac{a b}{\max \{a, b, 1\}}$ for each $a, b \in \mathbb{R}_{+}$, then for $\alpha \geq 1$, we have $a \diamond b \leq$ $(\max \{a, b\})^{\alpha}$.

\section{Main Results}

Now we prove some common fixed point theorems for weakly compatible mappings using binary operations in complete multiplicative metric spaces as follow:

Theorem 2.1. Let $(X, d)$ be a complete multiplicative metric space such that $\diamond$ satisfies a multiplicative $\alpha$-property with $\alpha>0$. Let $A, B, S$ and $T$ be mappings of $(X, d)$ into itself satisfying the following conditions

$$
T(X) \subset A(X), \quad S(X) \subset B(X)
$$

$$
d(S x, T y)
$$

$$
\begin{gathered}
\leq(d(A x, B y) \diamond d(A x, S x))^{k_{1}} \cdot(d(A x, B y) \diamond d(B y, T y))^{k_{2}} \\
\cdot\left(d(A x, B y) \diamond(d(A x, T y) \cdot d(S x, B y))^{\frac{1}{2}}\right)^{k_{3}}
\end{gathered}
$$

for all $x, y \in X$, where $k_{1}, k_{2}, k_{3}>0$ and $0<\alpha\left(k_{1}+k_{2}+k_{3}\right)<1$.

Assume that

$\left(C_{3}\right)$ the pairs $A, S$ and $B, T$ are weakly compatible, 
$\left(C_{4}\right)$ one of the subspace $A(X)$ or $B(X)$ or $S(X)$ or $T(X)$ is complete.

Then $A, B, S$ and $T$ have a unique common fixed point in $X$.

Proof. Let $x_{0} \in X$ be an arbitrary point. Since $S(X) \subset B(X)$, for $x_{0} \in X$, there exists $x_{1} \in X$ such that $S x_{0}=B x_{1}=y_{0}$. Now for this point $x_{1}$ there exists $x_{2} \in X$ such that $T x_{1}=A x_{2}=y_{1}$. Continuing in this manner, we can inductively define a sequence $\left\{y_{n}\right\}$ such that

$$
S x_{2 n}=B x_{2 n+1}=y_{2 n} ; \quad T x_{2 n+1}=A x_{2 n+2}=y_{2 n+1}
$$

for $n=0,1,2, \cdots$.

Now we prove $\left\{y_{n}\right\}$ is a Cauchy sequence in $X$. Using $\left(C_{2}\right)$, we have

$$
\begin{aligned}
& d\left(y_{2 n}, y_{2 n+1}\right) \\
&=d\left.S x_{2 n}, T x_{2 n+1}\right) \\
& \leq(\left.d\left(A x_{2 n}, B x_{2 n+1}\right) \diamond d\left(A x_{2 n}, S x_{2 n}\right)\right)^{k_{1}} \\
& \cdot\left(d\left(A x_{2 n}, B x_{2 n+1}\right) \diamond d\left(B x_{2 n+1}, T x_{2 n+1}\right)\right)^{k_{2}} \\
& \quad\left(d\left(A x_{2 n}, B x_{2 n+1}\right) \diamond\left(d\left(A x_{2 n}, T x_{2 n+1}\right) \cdot d\left(S x_{2 n}, B x_{2 n+1}\right)\right)^{\frac{1}{2}}\right)^{k_{3}} \\
&=\left(d\left(y_{2 n-1}, y_{2 n}\right) \diamond d\left(y_{2 n-1}, y_{2 n}\right)\right)^{k_{1}} \cdot\left(d\left(y_{2 n-1}, y_{2 n}\right) \diamond d\left(y_{2 n}, y_{2 n+1}\right)\right)^{k_{2}} \\
& \quad \cdot\left(d\left(y_{2 n-1}, y_{2 n}\right) \diamond\left(d\left(y_{2 n-1}, y_{2 n+1}\right) \cdot d\left(y_{2 n}, y_{2 n}\right)\right)^{\frac{1}{2}}\right)^{k_{3}} .
\end{aligned}
$$

Let $d_{n}=d\left(y_{n}, y_{n+1}\right)$. Then

$$
\begin{aligned}
d_{2 n} \leq & \left(d_{2 n-1} \diamond d_{2 n-1}\right)^{k_{1}} \cdot\left(d_{2 n-1} \diamond d_{2 n}\right)^{k_{2}} \cdot\left(d_{2 n-1} \diamond\left(d_{2 n-1} \cdot d_{2 n}\right)^{\frac{1}{2}}\right)^{k_{3}} \\
\leq & \left(d_{2 n-1}^{\alpha}\right)^{k_{1}} \cdot\left(\left(\max \left\{d_{2 n-1}, d_{2 n}\right\}\right)^{\alpha}\right)^{k_{2}} \\
& \cdot\left(\left(\max \left\{d_{2 n-1},\left(d_{2 n-1} \cdot d_{2 n}\right)^{\frac{1}{2}}\right)\right\}^{\alpha}\right)^{k_{3}} .
\end{aligned}
$$

If $d_{2 n}>d_{2 n-1}$, then

$$
d_{2 n} \leq d_{2 n}^{\alpha\left(k_{1}+k_{2}+k_{3}\right)},
$$

which is a contradiction. Hence $d_{2 n} \leq d_{2 n-1}$ so, from inequality $\left(C_{2}\right)$ we have

$$
d_{2 n} \leq d_{2 n-1}^{k},
$$

where $k=\alpha\left(k_{1}+k_{2}+k_{3}\right)<1$.

Similarly, we have

$$
d_{n} \leq d_{n-1}^{k}<d_{n-2}^{k^{2}} \leq \cdots \leq d_{0}^{k^{n}}
$$


Let $m, n \in \mathbb{N}$ with $m>n$. Then we get

$$
\begin{aligned}
d\left(y_{n}, y_{m}\right) & \leq d\left(y_{n}, y_{n+1}\right) \cdots d\left(y_{m-1}, y_{m}\right) \\
& \leq d^{k^{n}}\left(y_{0}, y_{1}\right) \cdots d^{k^{m-1}}\left(y_{0}, y_{1}\right) \\
& \left.\leq d^{\frac{k^{n}}{1-k}}\left(y_{0}, y_{1}\right)\right) \rightarrow 1
\end{aligned}
$$

as $n \rightarrow \infty$. It follows that $\left\{y_{n}\right\}$ is a multiplicative Cauchy sequence. Since $X$ is complete so $\left\{y_{n}\right\} \rightarrow z \in X$. Therefore, subsequences $\left\{S x_{2 n}\right\},\left\{B x_{2 n+1}\right\}$, $\left\{A x_{2 n}\right\}$ and $\left\{T x_{2 n+1}\right\}$ also converge to $z \in X$.

Now suppose that $A(X)$ is complete. Then there exists $w \in X$ such that $A w=z$.

Now, we claim that $S w=z$. Let $S w \neq z$. On putting $x=w$ and $y=x_{2 n+1}$ in inequality $\left(C_{2}\right)$, we get

$$
\begin{aligned}
& d\left(S w, y_{2 n+1}\right) \\
& =d\left(S w, T x_{2 n+1}\right) \\
& \leq\left(d\left(A w, B x_{2 n+1}\right) \diamond d(A w, S w)\right)^{k_{1}} \\
& \quad \cdot\left(d\left(A w, B x_{2 n+1}\right) \diamond d\left(B x_{2 n+1}, T x_{2 n+1}\right)\right)^{k_{2}} \\
& \quad \cdot\left(d\left(A w, B x_{2 n+1}\right) \diamond\left(d\left(A w, T x_{2 n+1}\right) \cdot d\left(S w, B x_{2 n+1}\right)\right)^{\frac{1}{2}}\right)^{k_{3}} .
\end{aligned}
$$

As $n \rightarrow \infty$, we have

$$
\begin{aligned}
d(S w, z) \leq & (d(z, z) \diamond d(z, S w))^{k_{1}} \cdot(d(z, z) \diamond d(z, z))^{k_{2}} \\
& \cdot\left(d(z, z) \diamond(d(z, z) \cdot d(S w, z))^{\frac{1}{2}}\right)^{k_{3}} \\
\leq & \left((\max \{1, d(z, S w)\})^{\alpha}\right)^{k_{1}} \cdot\left((\max \{1,1\})^{\alpha}\right)^{k_{2}} \\
& \cdot\left(\left(\max \left\{1, d^{\frac{1}{2}}(S w, z)\right\}\right)^{\alpha}\right)^{k_{3}} \\
= & d^{\alpha\left(k_{1}+\frac{k_{3}}{2}\right)}(S w, z),
\end{aligned}
$$

which is a contradiction. Hence $S w=z$. This implies $z=S w=A w$. Therefore, $w$ is a coincidence point of $A$ and $S$. Since $z=S w \in S(X) \subset B(X)$, there exists $v \in X$ such that $z=B v$.

Next, we claim that $T v=z$. Let $T v \neq z$. On putting $x=x_{2 n}$ and $y=v$ in inequality $\left(C_{2}\right)$, we have

$$
\begin{aligned}
& d\left(S x_{2 n}, T v\right) \\
& \leq\left(d\left(A x_{2 n}, B v\right) \diamond d\left(A x_{2 n}, S x_{2 n}\right)\right)^{k_{1}} \cdot\left(d\left(A x_{2 n}, B v\right) \diamond d(B v, T v)\right) k_{2} \\
& \cdot\left(d\left(A x_{2 n}, B v\right) \diamond\left(d\left(A x_{2 n}, T v\right) \cdot d\left(S x_{2 n}, B v\right)\right)^{\frac{1}{2}}\right)^{k_{3}} .
\end{aligned}
$$


As $n \rightarrow \infty$, we have

$$
\begin{aligned}
d(z, T v) \leq(d(z, z) \diamond d(z, z))^{k_{1}} \cdot(d(z, z) \diamond d(z, T v))^{k_{2}} \\
\cdot\left(d(z, z) \diamond(d(z, T v) \cdot d(z, z))^{\frac{1}{2}}\right)^{k_{3}} \\
\leq\left((\max \{1,1\})^{\alpha}\right)^{k_{1}} \cdot\left((\max \{1, d(T v, z)\})^{\alpha}\right)^{k_{2}} \\
\quad \cdot\left(\left(\max \left\{1, d^{\frac{1}{2}}(z, T v)\right\}\right)^{\alpha}\right)^{k_{3}} \\
=d^{\alpha\left(k_{2}+\frac{k_{3}}{2}\right)}(z, T v)
\end{aligned}
$$

which is a contradiction, therefore, $z=T v=B v$. Hence $v$ is a coincidence point of $B$ and $T$. Since the pairs $A, S$ and $B, T$ are weakly compatible, we have

$$
S z=S(A w)=A(S w)=A z
$$

and

$$
T z=T(B v)=B(T v)=B z .
$$

Next, we claim that $S z=z$. Let $S z \neq z$. Then using inequality $\left(C_{2}\right)$ and on putting $x=z$ and $y=x_{2 n+1}$, we have

$$
\begin{aligned}
& d\left(S z, T x_{2 n+1}\right) \\
& \leq\left(d\left(A z, B x_{2 n+1}\right) \diamond d(A z, S z)\right)^{k_{1}} \\
& \quad \cdot\left(d\left(A z, B x_{2 n+1}\right) \diamond d\left(B x_{2 n+1}, T x_{2 n+1}\right)\right)^{k_{2}} \\
& \quad \cdot\left(d\left(A z, B x_{2 n+1}\right) \diamond\left(d\left(A z, T x_{2 n+1}\right) \cdot d\left(S z, B x_{2 n+1}\right)\right)^{\frac{1}{2}}\right)^{k_{3}} .
\end{aligned}
$$

Letting $n \rightarrow \infty$, we have

$$
\begin{aligned}
d(S z, z) \leq & (d(S z, z) \diamond 1)^{k_{1}} \cdot(d(S z, z) \diamond d(z, z))^{k_{2}} \\
& \cdot\left(d(S z, z) \diamond(d(S z, z) \cdot d(S z, z))^{\frac{1}{2}}\right)^{k_{3}} \\
\leq & \left((\max \{d(S z, z), 1\})^{\alpha}\right)^{k_{1}} \cdot\left((\max \{d(S z, z), 1\})^{\alpha}\right)^{k_{2}} \\
& \cdot\left((\max \{d(S z, z), d(S z, z)\})^{\alpha}\right)^{k_{3}} \\
= & d^{\alpha\left(k_{1}+k_{2}+k_{3}\right)}(S z, z)
\end{aligned}
$$

which is a contradiction. So, we have $S z=z$ and hence we have $S z=A z=z$.

Finally, we claim that $T z=z$. Let $T z \neq z$. Then on putting $x=x_{2 n}$ and $y=z$ in inequality $\left(C_{2}\right)$, we have

$$
\begin{aligned}
d\left(S x_{2 n}, T z\right) \leq( & \left.d\left(A x_{2 n}, B z\right) \diamond d\left(A x_{2 n}, S x_{2 n}\right)\right)^{k_{1}} \\
\cdot & \left(d\left(A x_{2 n}, B z\right) \diamond d(B z, T z)\right)^{k_{2}} \\
\cdot & \left(d\left(A x_{2 n}, B z\right) \diamond\left(d\left(A x_{2 n}, T z\right) \cdot d\left(S x_{2 n}, B z\right)\right)^{\frac{1}{2}}\right)^{k_{3}} .
\end{aligned}
$$


Letting $n \rightarrow \infty$, we have

$$
\begin{aligned}
d(z, T z) \leq & (d(z, T z) \diamond 1)^{k_{1}} \cdot(d(z, T z) \diamond 1)^{k_{2}} \\
& \cdot\left(d(z, T z) \diamond(d(z, T z) \cdot d(z, T z))^{\frac{1}{2}}\right)^{k_{3}} \\
\leq & \left((\max \{d(z, T z), 1\})^{\alpha}\right)^{k_{1}} \cdot\left((\max \{d(T z, z), 1\})^{\alpha}\right)^{k_{2}} \\
& \cdot\left((\max \{d(T z, z), d(T z, z)\})^{\alpha}\right)^{k_{3}} \\
= & d^{\alpha\left(k_{1}+k_{2}+k_{3}\right)}(T z, z)
\end{aligned}
$$

which is a contradiction, therefore, we have $T z=z$ and hence we conclude that $T z=B z=z$. Therefore, $z$ is a common fixed point of $A, B, S$ and $T$.

Similarly we can complete the proof for cases in which $B(X)$ or $S(X)$ or $T(X)$ is complete

Uniqueness can easily follows from inequality $\left(C_{2}\right)$. This completes the proof.

In Theorem 2.1, if we put $S=T$, then we obtain the following corollary.

Corollary 2.2. Let $(X, d)$ be a complete multiplicative metric space such that $\diamond$ satisfies a multiplicative $\alpha$-property with $\alpha>0$. Let $A, B$ and $S$ be mappings of $(X, d)$ into itself satisfying the following conditions

$$
S(X) \subset A(X), \quad S(X) \subset B(X),
$$

$$
\begin{gathered}
d(S x, S y) \\
\leq(d(A x, B y) \diamond d(A x, S x))^{k_{1}} \cdot(d(A x, B y) \diamond d(B y, S y))^{k_{2}} \\
\cdot\left(d(A x, B y) \diamond(d(A x, S y) \cdot d(S x, B y))^{\frac{1}{2}}\right)^{k_{3}}
\end{gathered}
$$

for all $x, y \in X$, where $k_{1}, k_{2}, k_{3}>0$ and $0<\alpha\left(k_{1}+k_{2}+k_{3}\right)<1$.

Assume that

$\left(C_{7}\right)$ the pairs $A, S$ and $B, S$ are weakly compatible,

$\left(C_{8}\right)$ one of the subspaces $A(X)$ or $B(X)$ or $S(X)$ is complete.

Then $A, B$ and $S$ have a unique common fixed point in $X$.

In Theorem 2.1, if we put $A=B=I$, then we obtain the following corollary.

Corollary 2.3. Let $(X, d)$ be a complete multiplicative metric space such that $\diamond$ satisfies a multiplicative $\alpha$-property with $\alpha>0$. Let $S$ and $T$ be mappings of $(X, d)$ into itself satisfying the following conditions

$$
\begin{gathered}
d(S x, T y) \leq(d(x, y) \diamond d(x, S x))^{k_{1}} \cdot(d(x, y) \diamond d(y, T y))^{k_{2}} \\
\cdot\left(d(x, y) \diamond(d(x, T y) \cdot d(S x, y))^{\frac{1}{2}}\right)^{k_{3}}
\end{gathered}
$$


for all $x, y \in X$, where $k_{1}, k_{2}, k_{3}>0$ and $0<\alpha\left(k_{1}+k_{2}+k_{3}\right)<1$.

Assume that

$\left(C_{10}\right)$ one of the subspaces $S(X)$ or $T(X)$ is complete.

Then $S$ and $T$ have a unique common fixed point in $X$.

Now we prove common fixed point theorems for weakly compatible mappings in multiplicative metric spaces without completeness of $X$ as follow:

Theorem 2.4. Let $(X, d)$ be a multiplicative metric space such that $\diamond$ satisfies a multiplicative $\alpha$-property with $\alpha>0$. Let $A, B, S$ and $T$ be mappings of $(X, d)$ into itself satisfying the conditions $\left(C_{1}\right)-\left(C_{4}\right)$. Then $A, B, S$ and $T$ have a unique common fixed point.

Proof. From the proofs of Theorem 2.1, $\left\{y_{n}\right\}$ is a multiplicative Cauchy sequence. Suppose that $A(X)$ is complete. Then there exists $u \in A(X)$ such that

$$
y_{2 n+1}=A x_{2 n+2}=T x_{2 n+1} \rightarrow u \quad \text { as } n \rightarrow \infty .
$$

Consequently, we can find $v \in X$ such that $A v=u$. Further a multiplicative Cauchy sequence $\left\{y_{n}\right\}$ has a convergent subsequence $\left\{y_{2 n+1}\right\}$, therefore, the sequence $\left\{y_{n}\right\}$ also converges and hence a subsequence $\left\{y_{2 n}\right\}$ also converges. Thus we have

$$
y_{2 n}=B x_{2 n+1}=S x_{2 n} \rightarrow u \quad \text { as } n \rightarrow \infty .
$$

Now, we claim $S v=u$. Let $S v \neq u$. Then putting $x=v$ and $y=x_{2 n+1}$ in inequality $\left(C_{2}\right)$, we get

$$
\begin{aligned}
& d\left(S v, y_{2 n+1}\right) \\
& =d\left(S v, T x_{2 n+1}\right) \\
& \leq\left(d\left(A v, B x_{2 n+1}\right) \diamond d(A v, S v)\right)^{k_{1}} \\
& \quad \cdot\left(d\left(A v, B x_{2 n+1}\right) \diamond d\left(B x_{2 n+1}, T x_{2 n+1}\right)\right)^{k_{2}} \\
& \quad \cdot\left(d\left(A v, B x_{2 n+1}\right) \diamond\left(d\left(A v, T x_{2 n+1}\right) \cdot d\left(S v, B x_{2 n+1}\right)\right)^{\frac{1}{2}}\right)^{k_{3}} .
\end{aligned}
$$

Letting $n \rightarrow \infty$, we have

$$
\begin{aligned}
d(S v, u) \leq( & d(u, u) \diamond d(u, S v))^{k_{1}} \cdot(d(u, u) \diamond d(u, u))^{k_{2}} \\
& \cdot\left(d(u, u) \diamond(d(u, u) \cdot d(S v, u))^{\frac{1}{2}}\right)^{k_{3}} \\
\leq & \left((\max \{1, d(u, S v)\})^{\alpha}\right)^{k_{1}} \cdot\left((\max \{1,1\})^{\alpha}\right)^{k_{2}} \\
& \cdot\left(\left(\max \left\{1, d^{\frac{1}{2}}(u, S v)\right\}\right)^{\alpha}\right)^{k_{3}} \\
= & d^{\alpha\left(k_{1}+\frac{k_{3}}{2}\right)}(u, S v)
\end{aligned}
$$


which is a contradiction. Hence $S v=u$ and hence $u=A v=S v$, that is $v$ is a coincidence point of $A$ and $S$.

Since $u=S v \in S(X) \subset B(X)$, there exists $w \in X$ such that $u=B w$.

Next, we claim $T w=u$. Let $T w \neq u$. Then on putting $x=v$ and $y=w$ in inequality $\left(C_{2}\right)$, we have

$$
\begin{aligned}
& d(u, T w) \\
& =d(S v, T w) \\
& \leq(d(A v, B w) \diamond d(A v, S v))^{k_{1}} \cdot(d(A v, B w) \diamond d(B w, T w))^{k_{2}} \\
& \quad \cdot\left(d(A v, B w) \diamond(d(A v, T w) \cdot d(S v, B w))^{\frac{1}{2}}\right)^{k_{3}} \\
& =d(u, u) \diamond d(u, u))^{k_{1}} \cdot(d(u, u) \diamond d(u, T w))^{k_{2}} \\
& \quad \cdot\left(d(u, u) \diamond(d(u, T w) \cdot d(u, u))^{\frac{1}{2}}\right)^{k_{3}} \\
& \leq\left((\max \{1,1\})^{\alpha}\right)^{k_{1}} \cdot\left((\max \{1, d(u, T w)\})^{\alpha}\right)^{k_{2}} \\
& \quad \cdot\left(\left(\max \left\{1, d^{\frac{1}{2}}(u, T w)\right\}\right)^{\alpha}\right)^{k_{3}} \\
& =d^{\alpha\left(k_{2}+\frac{k_{3}}{2}\right)}(u, T w),
\end{aligned}
$$

which is a contradiction, which implies that $u=T w$ and hence $u=B w=T w$, that is, $w$ is a coincidence point of $B$ and $T$. Since the pairs $A, S$ and $B, T$ are weakly compatible, we have

$$
S u=S(A v)=A(S v)=A u=w_{1} \quad(\text { say })
$$

and

$$
T u=T(B w)=B(T w)=B u=w_{2} \quad \text { (say). }
$$

From inequality $\left(C_{2}\right)$, we have

$$
\begin{aligned}
d\left(w_{1}, w_{2}\right)= & d(S u, T u) \\
\leq & (d(A u, B u) \diamond d(A u, S u))^{k_{1}} \cdot(d(A u, B u) \diamond d(B u, T u))^{k_{2}} \\
& \cdot\left(d(A u, B u) \diamond(d(A u, T u) \cdot d(S u, B u))^{\frac{1}{2}}\right)^{k_{3}} \\
= & \left(d\left(w_{1}, w_{2}\right) \diamond d\left(w_{1}, w_{1}\right)\right)^{k_{1}} \cdot\left(d\left(w_{1}, w_{2}\right) \diamond d\left(w_{2}, w_{2}\right)\right)^{k_{2}} \\
& \cdot\left(d\left(w_{1}, w_{2}\right) \diamond\left(d\left(w_{1}, w_{2}\right) \cdot d\left(w_{1}, w_{2}\right)\right)^{\frac{1}{2}}\right)^{k_{3}} \\
\leq & \left(\left(\max \left\{d\left(w_{1}, w_{2}\right), 1\right\}\right)^{\alpha}\right)^{k_{1}} \cdot\left(\left(\max \left\{d\left(w_{1}, w_{2}\right), 1\right\}\right)^{\alpha}\right)^{k_{2}} \\
& \quad \cdot\left(\left(\max \left\{d\left(w_{1}, w_{2}\right), d\left(w_{1}, w_{2}\right)\right\}\right)^{\alpha}\right)^{k_{3}} \\
= & d^{\alpha\left(k_{1}+k_{2}+k_{3}\right)}\left(w_{1}, w_{2}\right),
\end{aligned}
$$


which is a contradiction, that is, $w_{1}=w_{2}$. Therefore, we have $S u=A u=T u=$ $B u$

Again using inequality $\left(C_{2}\right)$, we have

$$
\begin{aligned}
d(S v, T u) \leq( & d(A v, B u) \diamond d(A v, S v))^{k_{1}} \cdot(d(A v, B u) \diamond d(B u, T u))^{k_{2}} \\
& \cdot\left(d(A v, B u) \diamond(d(A v, T u) \cdot d(S v, B u))^{\frac{1}{2}}\right)^{k_{3}} \\
= & (d(S v, T u) \diamond d(A v, S v))^{k_{1}} \cdot(d(S v, T u) \diamond d(T u, T u))^{k_{2}} \\
& \cdot\left(d(S v, T u) \diamond(d(S v, T u) \cdot d(S v, T u))^{\frac{1}{2}}\right)^{k_{3}} \\
\leq & \left((\max \{d(S v, T u), 1\})^{\alpha}\right)^{k_{1}} \cdot\left((\max \{d(S v, T u), 1\})^{\alpha}\right)^{k_{2}} \\
& \cdot\left((\max \{d(S v, T u), d(S v, T u)\})^{\alpha}\right)^{k_{3}} \\
= & d^{\alpha\left(k_{1}+k_{2}+k_{3}\right)}(S v, T u),
\end{aligned}
$$

which is a contradiction, this implies that $S v=T u$, that is, $u=T u$. Therefore $u$ is a common fixed point of $A, B, S$ and $T$.

Similarly we can complete the proof for cases in which $B(X)$ or $S(X)$ or $T(X)$ is complete

Uniqueness can easily follows from inequality $\left(C_{2}\right)$. This completes the proof.

In Theorem 2.4, if we put $S=T$, then we obtain the following corollary.

Corollary 2.5. Let $(X, d)$ be a multiplicative metric space such that $\diamond$ satisfies a multiplicative $\alpha$-property with $\alpha>0$. Let $A, B$ and $S$ be mappings of $(X, d)$ into itself satisfying the conditions $\left(C_{5}\right)-\left(C_{8}\right)$. Then $A, B$ and $S$ have a unique common fixed point in $X$.

In Theorem 2.4, if we put $A=B=I$, then we obtain the following corollary.

Corollary 2.6. Let $(X, d)$ be a multiplicative metric space such that $\diamond$ satisfies a multiplicative $\alpha$-property with $\alpha>0$. Let $S$ and $T$ be mappings of $(X, d)$ into itself satisfying the conditions $\left(C_{9}\right)$ and $\left(C_{10}\right)$. Then $S$ and $T$ have a unique common fixed point in $X$.

\section{References}

[1] M. Abbas, B. Ali, Y.I. Suleiman, Common fixed points of locally contractive mappings in multiplicative metric spaces with application, Int. J. Math. Math. Sci., 2015 (2015), Article ID 218683, 7 pages. doi: 10.1155/2015/218683.

[2] M.A. Ahmed, Common fixed point theorems for weakly compatible mappings, Rocky Mountain J. Math., 33 (2003), 1189-1203.doi: 10.1216/rmjm/1181075457 
[3] A.E. Bashirov, E.M. Kurplnara, A. Ozyapici, Multiplicative calculus and its applications, J. Math. Anal. Appl., 337 (2008), 36-48. doi: 10.1016/j.jmaa.2007.03.081

[4] R. Chugh, S. Kumar, Common fixed points for weakly compatible maps, Proc. Indian Acad. Sci. Math. Sci., 111 (2001), 241-247.

[5] Lj.B. Ćirić, J.S. Ume, Some common fixed point theorems for weakly compatible mappings, J. Math. Anal. Appl., 314 (2006), 488-499. doi: 10.1016/j.jmaa.2005.04.007

[6] F. Gu, L.M. Cui, Y.H. Wu, Some fixed point theorems for new contractive type mappings, J. Qiqihar Univ., 19 (2013), 85-89.

[7] X. He, M. Song, D. Chen, Common fixed points for weak commutative mappings on a multiplicative metric space, Fixed Point Theory Appl., 48 (2014), 9 pages. doi: $10.1186 / 1687-1812-2014-48$

[8] G. Jungck, Common fixed points for noncontinuous nonself maps on nonmetric spaces, Far East J. Math. Sci., 4 (1996), 199-215.

[9] M. Özavsar, A.C. Çevikel, Fixed points of multiplicative contraction mappings on multiplicative metric spaces, arXiv:1205.5131v1 [math.GM], 2012.

[10] V. Popa, A general fixed point theorem for four weakly compatible mappings satisfying an implicit relation, Filomat, 19 (2005), 45-51.

[11] M. Sarwar, R. Badshah-e, Some unique fixed point theorems in multiplicative metric space, arXiv:1410.3384v2 [math.GM], 2014.

[12] S. Sedghi, N. Shobe, Common fixed point theorems for four mappings in complete metric spaces, Bull. Iranian Math. Soc., 33 (2007), 37-47. 
